\title{
Splintering and Inertia in Network Industries*
}

\author{
Tobias Kretschmer \\ ICE, University of Munich and $C E P^{\dagger}$
}

February 2007

\begin{abstract}
We analyze a stylized game of technology adoption with network effects and two new technologies. Potential adopters can adopt early, late, or not at all. We show that one of the reasons for the failure of new technologies can be the presence of multiple incompatible variants of that technology. An adopter's individual incentives to adopt are lower with two technologies than with one. Turning to aggregate expected welfare, we find that two active technologies may be welfare-improving. JEL: L1, O3. Keywords: Technology Adoption, Splintering, Network Effects, Installed Base.
\end{abstract}

\section{Introduction}

When a new technology is introduced, potential adopters often face a choice between different variants of the technology. With network effects, this can

${ }^{*}$ I thank Luís Cabral, Paul Geroski, Carmen Matutes, Pierre Régibeau, Peter Zemsky, editor Yeon-Koo Che, an anonymous referee and seminar audiences at LSE, LBS, East Anglia, Zurich, Rotterdam, Amsterdam, Münster, ECARES, Leuven, INSEAD, IAE Barcelona and various conferences and workshops for helpful discussions and comments, and the Institut d'Analisi Economico and INSEAD for their hospitality. Financial support from London Business School and the ESRC is gratefully acknowledged. The usual disclaimer applies.

$\dagger$ Institute for Communication Economics, Munich School of Management, University of Munich, Schackstr. 4/III, D - 80539 Munich, Germany. email: t.kretschmer@lmu.de. 
lead to 'splintering' (Farrell and Klemperer, forthcoming) among technologies. For example, Quadraphonic sound came in two different versions, discrete and matrix, which deterred many adopters from choosing either of them for fear of ending up with the unsuccessful one (Postrel, 1990). Similarly, when the DVD faced competition for the digital video market by DivX, consumers and providers of digital video recognized the danger of a fragmented market with both technologies attracting a number of consumers and content providers, leading to lower network effects overall (Dranove and Gandal, 2003). In both these cases (and numerous others), there is a tradeoff between the increased diversity through multiple (differentiated) versions of a new technology on the one hand and the potential for coordination failure through splintering among different variants on the other. In this paper, we explicitly model this tradeoff to study the impact of multiple new technologies on the likelihood of transition to a new standard and the welfare implications of multiple new technologies entering.

We analyze a stylized game of technology adoption with network effects and two new technologies. In a two-period game with two heterogeneous adopters and imperfect information, we study the emergence of a Bandwagon Equilibrium, i.e. an equilibrium in which some types of adopters move early and others mimic the observed behaviour in the second period. This setup allows us to model a situation where adopters have different inherent (standalone) preferences, but net payoffs are interdependent due to network effects, creating the possibility of coordination failures. With one new technology, there is only one coordination problem - old versus new - but this problem is largely solved dynamically (Farrell and Saloner, 1985) with high-value adopters initiating a bandwagon and intermediate adopters following. Having a second (independently drawn) new technology clearly increases welfare if adoption can be centrally coordinated, but makes decentralized coordination more difficult, as there are now two coordination problems - old versus new (inertia), and new versus new (splintering). The latter coordination problem can aggravate the former, since high valuation adopters are more willing to wait to avoid adopting an orphaned technology, thus reducing their incentives to initiate a bandwagon. Therefore, overall welfare can fall with two technologies as splintering implies a loss in network effects.

The literature on the 'old versus new' coordination problem with network effects considers either exogenous (Katz and Shapiro, 1986, Choi, 1994, 1997, Choi and Thum, 1998) or endogenous timing of adoption (Farrell and Saloner, 1985). The former find that early adopters impose their preferences on the 
market, exerting a negative (forward) externality on latecomers. However, these papers do not capture the possibility that early adopters may be put off from initiating a bandwagon by the uncertainty over the preferences of later adopters. Models with endogeneous timing explicitly allow for this possibility, although only with one new technology (Farrell and Saloner, 1985). In single-technology models, splintering between different variants is not a risk, which rules out analysis of some important cases such as different flavours of Unix (Saloner, 1990), Quadraphonic sound (Postrel, 1990), or 56k modems (Augereau, Greenstein and Rysman, 2004). The 'new versus new' coordination problem among adopters has been studied in the literature on standard battles, although mostly with exogenous timing (Arthur, 1989, Cabral and Kretschmer, 2007). Anecdotal and empirical evidence (Postrel, 1990, Koski, 1999) suggests however that adoption incentives change over the different stages of a standards battle.

Our paper incorporates the welfare effects of delayed adoption and the opportunity cost of unrealized network benefits. It is the first to formally analyze the possibility of splintering and its effect on adoption incentives and timing by combining the coordination problems of 'old versus new' and 'new versus new' in a single framework.

The paper is structured as follows. Section 2 introduces the model and our equilibrium concept. Section 3 discusses equilibrium parameter ranges and behaviour for two technologies with independent values, and considers a single new technology and two technologies with common values. We compare the one-technology and two-technology cases in Section 4. We discuss limitations and possible extensions in Section 5 and conclude in Section 6.

\section{The Model}

- Setup of the game. Consider two ex-ante symmetric agents $i, j$ with private information about their preferences. Two new technologies $x$ and $y$ are supplied at marginal cost $c$. Switching can take place in periods $t=1,2$ or not at all, which implies remaining with the incumbent technology. Adoption decisions are observed at the end of each period. We ignore discounting and flow benefits. ${ }^{1}$ Adoption is irreversible and adopters will use at most one

\footnotetext{
${ }^{1}$ Discounting and/or flow benefits would generate the standard incentives to hasten adoption. Omitting these factors is in line with other work on coordination issues between adopters (see, e.g. Farrell and Saloner, 1985, and Choi, 1997).
} 
of the technologies.

Realized Payoffs. We normalize utility from the incumbent technology to $0 .^{2}$ Net utility for new technology $x$ consists of the standalone value $a^{x}$, (common) network benefits $b$, and adoption cost $c .^{3}$ If an adopter adopts new technology $x$, her realized utility at the end of the game will be

$$
u_{i}^{x}\left(a_{i}^{x}, b, c, z_{j}^{x}\right)=a_{i}^{x}+b z_{j}^{x}-c,
$$

where $z_{j}^{x}=1$ if player $j$ has adopted $x$ and $z_{j}^{x}=0$ otherwise. The expression for $y$ is formed analogously. Network benefits only accrue if both adopters choose the same technology.

Valuations and Parameter Restrictions. Standalone valuations for technology $x$ are $a^{x} \in\{h, l, 0\}$, where $h>l>0$, and analogously for $y{ }^{4}$ The realizations are private information, but it is common knowledge that they are drawn with probabilities $p, q$, and $1-p-q$, respectively. An adopter's type is characterized by the valuation pair denoted $\mathbf{a}=\left(a^{x} a^{y}\right) \in$ $\{(h h),(h l),(l h),(h 0),(0 h),(l l),(l 0),(0 l),(00)\}$. We write the probability of an adopter being of a specific type a as $\phi_{\mathbf{a}}$. Denote by $a_{\max }\left(a_{\min }\right)$ an adopter's weakly highest (lowest) valuation for the technologies. Figure 1 illustrates the valuation pairs and attaches labels to them. A type's maximum valuation $a_{\max }$ determines whether she is labelled an 'enthusiast', a 'follower', or an 'intransigent'. The difference between maximum and minimum valuation, $a_{\max }-a_{\min }$, on the other hand, indicates whether she is 'uniform' (if $a_{\max }-a_{\min }=0$ ), 'biased' (if $0<a_{\max }-a_{\min }<h$ ), or 'stubborn' (if $\left.a_{\max }-a_{\min }=h\right){ }^{5}$

Assume $b-c<0,{ }^{6}$ and $l+b-c>0$, so that an $l$-type would adopt a technology that carries the full network benefit (and so would $h$-types).

\footnotetext{
${ }^{2}$ This assumes that adopters switching does not diminish the value of the old technology, for instance because there is a large installed base beyond the two adopters we model or there is a wide variety of complementary products for the old technology. Relaxing this assumption would strengthen the tendency to follow a bandwagon.

${ }^{3}$ Differences in adoption costs across agents are captured by differences in valuations for the new technology, and the assumption of common $b$ is standard in the literature.

${ }^{4}$ We omit the subscript $i, j$ where there is no ambiguity.

${ }^{5}$ We assume that 'uniform' adopters have a weak preference for one of the technologies, i.e. $a_{\max }=a_{\min }+\varepsilon$. Uniform adopters have an even chance of weakly preferring technology $x$ or $y$.

${ }^{6}$ Farrell and Saloner (1985) show that a bandwagon will only emerge if some types,
} 


\begin{tabular}{|c|c|c|c|}
\cline { 2 - 4 }$a^{y}=h$ & $\begin{array}{c}\text { stubborn } \\
\text { enthusiast }\end{array}$ & $\begin{array}{c}\text { biased } \\
\text { enthusiast }\end{array}$ & $\begin{array}{c}\text { uniform } \\
\text { enthusiast }\end{array}$ \\
\cline { 2 - 4 }$a^{y}=l$ & $\begin{array}{c}\text { biased } \\
\text { follower }\end{array}$ & $\begin{array}{c}\text { uniform } \\
\text { follower }\end{array}$ & $\begin{array}{c}\text { biased } \\
\text { enthusiast }\end{array}$ \\
\cline { 2 - 4 }$a^{y}=0$ & intransigent & $\begin{array}{c}\text { biased } \\
\text { follower }\end{array}$ & $\begin{array}{c}\text { stubborn } \\
\text { enthusiast }\end{array}$ \\
\cline { 2 - 4 } & \multicolumn{2}{|c|}{$\begin{array}{c}a^{x}=0 \\
a^{x}=l\end{array}$}
\end{tabular}

Figure 1: Valuation pairs and adopter characterizations.

We can then focus on coordination issues among adopters and technologies. Further suppose that $h<l+b$, which limits our analysis to the most interesting case where adopters may have a modest preference for one of the technologies but prefer to be compatible with the other player. Relaxing this assumption (so that $h>l+b$ ) would in fact make analysis simpler (because equilibrium strategies of stubborn and biased enthusiasts would coincide), but less interesting.

Actions and Strategies. An adopter's strategy $\sigma_{\mathbf{a}}$ consists of her adoption probabilities at each node in the game. Adopters face a decision node at $t=1$ and in three subgames at $t=2$.

Start of the game: At $t=1$, adopt (weakly) preferred technology with probability $\alpha_{\mathbf{a}}$.

Subgame 1 (S1): At $t=2$ if there has been no adoption in 1 , adopt (weakly) preferred technology with probability $\beta_{\mathbf{a}}$.

Subgame 2 (S2): At $t=2$ if there has been adoption of one's preferred

intransigents in their terminology, would never adopt. Assuming that $b-c<0$ and the possibility of zero valuation ensures this in our model. 
technology in 1, adopt preferred technology with probability $\gamma_{\mathbf{a}}$.

Subgame 3 (S3): At $t=2$ if there has been adoption of one's less preferred technology in 1, adopt less preferred technology with probability $\delta_{\mathbf{a}}$.

An adopter's strategy for the entire game is denoted $\sigma_{\mathbf{a}}=\left\{\alpha_{\mathbf{a}}, \beta_{\mathbf{a}}, \gamma_{\mathbf{a}}, \delta_{\mathbf{a}}\right\} .{ }^{7}$ Note that if $\alpha_{\mathbf{a}}>0$, an adopter may start a bandwagon, and if $\gamma_{\mathbf{a}}=1$, the adopter is a 'bandwagon' adopter. ${ }^{8}$

Equilibrium. Every valuation pair a maps into an equilibrium strategy $\sigma_{\mathbf{a}}^{*}$. We solve for Perfect Bayesian Nash Equilibria in mixed strategies (PBNE). In other words, at every node, player $i$ 's action maximizes expected payoffs given current beliefs about $j$ 's valuations. We are interested in settings where transition to the new technology is not guaranteed, but possible, and where some (types of) adopters need to be induced to switch by observing prior adoption. We therefore concentrate on the parameter ranges in which there exist Bandwagon Equilibria:

Definition: A Bandwagon Equilibrium (BE) is a Perfect Bayesian Nash Equilibrium with a positive probability of adoption at $t=1$ and types that would only adopt at $t=2$ if there has been adoption at $t=1$.

This definition imposes restrictions on the values of $h, l, b$ and $c$ to obtain valuation vectors a such that some types of adopters have equilibrium strategies $\alpha_{\mathbf{a}}^{*}=\beta_{\mathbf{a}}^{*}=0, \gamma_{\mathbf{a}}^{*}=1$, and some have $\alpha_{\mathbf{a}}^{*}>0$. The former requires that some types have a valuation high enough to adopt if they are guaranteed network effects and low enough to keep them from adopting on their own. The latter restriction states that some types value a new technology highly enough to consider switching early to get a bandwagon started. We now derive conditions for a $B E$ to obtain.

\footnotetext{
${ }^{7}$ Adopting one's preferred technology in $S 3$ implies $a_{\max }>a_{\min }+b$. In this case, the equilibrium strategy would then be to adopt at $t=1\left(\alpha_{\mathbf{a}}^{*}=1\right)$, so we ignore this theoretical possibility for notational convenience.

${ }^{8}$ A "Bandwagon Strategy", in Farrell and Saloner's (1985) terminology, involves delaying adoption at $t=1$ and mimicking the other adopter's $t=1$ action at $t=2$. In our model, this need not occur because an early adopter may adopt the less preferred technology and will consequently not be followed (if $\delta_{\mathbf{a}}=0$ ). We stick to Farrell and Saloner's terminology however to describe the readiness to imitate adoption of one's own preferred technology.
} 


\section{Derivation of Equilibria}

Equilibrium analysis. We are interested in defining the parameter space $(h, l, p, q)$ that supports a $B E .^{9}$ Three forces determine an adopter's incentives to adopt early in a $B E$ - the likelihood of starting a bandwagon, the risk of splintering through simultaneous adoption, and the value of adopting the less preferred technology. To gain an intuitive understanding of our equilibrium consider the types with $a_{\max }=h$ : stubborn, biased, and uniform enthusiasts. For all enthusiasts, adopting early may result in orphaning (i.e. adopting without obtaining the network benefit) for two reasons - either no bandwagon emerges or the market has splintered.

The difference in adoption incentives across types comes from the value of subgame $S 3$ at $t=2$ - adopting the less preferred technology if adoption has taken place at $t=1$. A stubborn enthusiast derives zero utility from adopting her less preferred technology, while a biased enthusiast derives some, ${ }^{10}$ and a uniform enthusiast is indifferent between either of the technologies, as long as one of them gets adopted. The incentive to delay adoption is increasing in the valuation of the less preferred technology. For a stubborn enthusiast, waiting is never profitable - if standalone valuation and expected network benefits outweigh adoption costs, early adoption is always more likely to trigger bandwagon behaviour than waiting until $t=2$. Will a uniform enthusiast ever adopt early? A uniform enthusiast will weigh up the potential of starting a bandwagon by adopting at $t=1$ and the risk of splintering. If starting a bandwagon is important (if, for example, there is a high likelihood of the other adopter having $a_{\max }=l$ ), a uniform enthusiast may still want to adopt early. The tendency to adopt early or late will also be governed by expectations about the adoption times of other potential adopters to minimize the risk of splintering. Proposition 1a summarizes the properties of a $B E$ with two independent new technologies. ${ }^{11}$

\footnotetext{
${ }^{9}$ There are two other types of equilibria, coordination game and no-adoption equilibria. A coordination game equilibrium arises if all types with $a_{\max }=h, l$ adopt early (and 0types never adopt). This occurs for high standalone valuations $(l)$ and network benefits $(b)$ for low-valuation adopters. Conversely, an equilibrium with no adoption at all results from a low probability of being followed $(p, q)$, low standalone benefits $(h)$, and low network benefits $(b)$ for potential early adopters.

${ }^{10}$ In fact, a biased enthusiast still derives higher utility from adopting her less preferred technology than from adopting her preferred technology on her own since $l+b>h$.

${ }^{11}$ All proofs are in the Appendix.
} 
Proposition 1a: If valuations for two technologies are independently drawn, then for sufficiently high valuations for enthusiasts and sufficiently low valuations for followers, two BE may exist, a high-adoption and a lowadoption BE. For $h>\widehat{h}_{\text {high }}=c-\left(p+q-p q-\frac{\alpha_{h h}}{2} p^{2}\right) b$ and $l<\widehat{l}_{\text {high } 1}=$ $c-\left(\frac{q^{2}+2 q(1-p-q)}{2\left(1-2 p\left(1-\frac{p}{2}\right)\right)}\right) b$, a high-adoption BE exists where stubborn and biased enthusiasts adopt early with certainty, and uniform enthusiasts adopt their weakly preferred technology early with probability $\alpha_{h h}^{*}=\frac{\frac{3}{2} p^{2}-p q+q-p}{p^{2}}$. For $h>\widehat{h}_{\text {low }}=c-\left(p+q-\alpha_{\text {hl }} p q\right) b$ and $l<\widehat{l}_{\text {low }}=c-\left(\frac{2 p q+p^{2}+q^{2}+2 q(1-p-q)}{2(1-2 p(1-p-q))}\right) b, a$ low-adoption BE exists where stubborn enthusiasts adopt early with certainty, biased enthusiasts adopt early with probability $\alpha_{h l}^{*}=\frac{p(1-p-q)(l+b-h)-\left(\frac{p^{2}}{2}+q\right) b}{p q(l+b-h)+p q b}$ and uniform enthusiasts delay with certainty. In both BE, followers play a bandwagon strategy.

The mixing probabilities $\alpha_{h h}^{*}$ and $\alpha_{h l}^{*}$ are non-degenerate (i.e. $0<\alpha_{.}^{*}<1$ ) only for certain parameter ranges, as illustrated by Figure 2. If there is a high probability of the other adopter playing a bandwagon strategy, i.e. $a_{\max }=l$, even a uniform enthusiast will adopt early with certainty in order to start a bandwagon. This is the top left corner in the figure. As the ratio $\frac{p}{q}$ increases, the danger of splintering increases and an indifferent adopter will delay with positive probability. If $q$ decreases further, this effect increases and the likelihood of being followed by a bandwagon adopter decreases, thus resulting in mixing behaviour even by biased enthusiasts $(\mathbf{a}=(h l))$.

We now study two special cases of our basic model, first, a single new technology, and second two technologies with common values.

- Special case 1: Single new technology. This case resembles Farrell and Saloner (1985) with discrete valuations and is a special case of our model where $a^{y}=0 \quad \forall \mathbf{a}$. We use it as a benchmark to assess the effect of splintering, which does not exist in the single-technology case.

Proposition 1b: A BE in the one-technology case consists of enthusiasts adopting early with certainty and followers playing a bandwagon strategy.

The intuition behind this $B E$ is straightforward: If an adopter has a sufficiently high expectation of being followed given her standalone valuation, she will adopt at $t=1$. There is no incentive for an enthusiast to mix adoption times - if early adoption is profitable, it never pays to delay. 


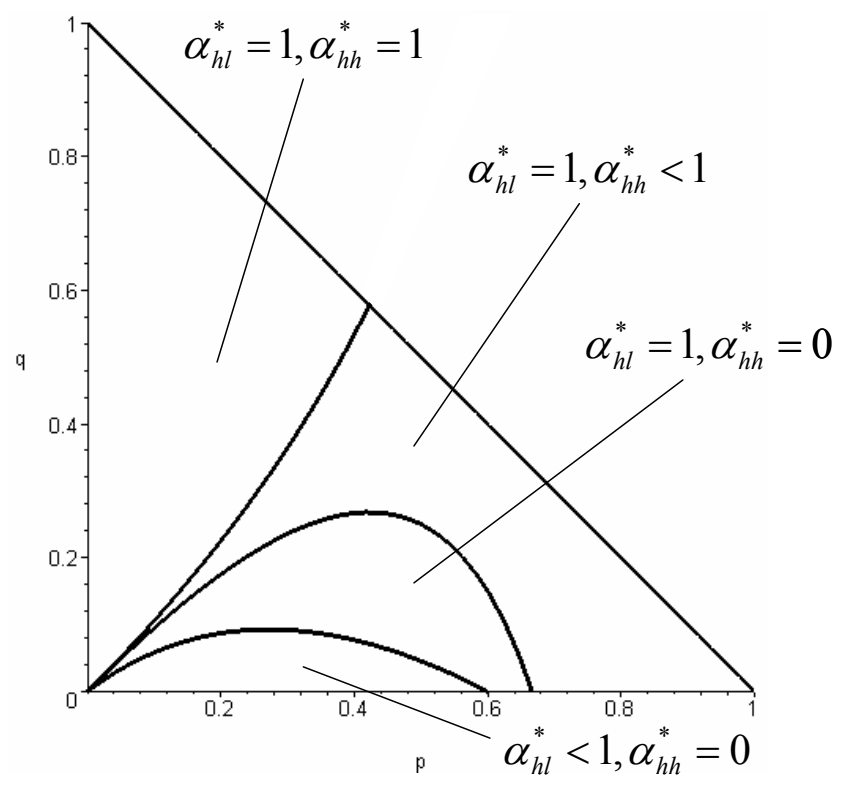

Figure 2: $p, q$ ranges for non-degenerate mixed strategies. (Note: For $\alpha_{h l}^{*}$, the following parameter values were used (in relation to $h$ ): $l=.875 h, b=.5 h$ )

- Special case 2: Perfect Substitutes. It is often argued that new technologies are more or less identical, i.e. they appeal to the same consumers. This can be modelled as $a_{\max }=a_{\min } \forall \mathbf{a}$. Suppose further that there is no focal point, i.e. adopters do not automatically coordinate on $x$ or $y$ if they are indifferent between the two. ${ }^{12}$ The $B E$ can then be characterized as follows.

Proposition 1c: The unique BE in the case of two common value technologies involves enthusiasts adopting early with probability $\alpha_{h}^{*}=\frac{1}{2}+\frac{q}{p}$ and followers plaing a bandwagon strategy.

This result emphasizes the effects of splintering. In the one-technology scenario there is only the risk of the other adopter being an intransigent (with valuation $\mathbf{a}_{\max }=0$ ), but there is now the risk of both moving simulta-

\footnotetext{
${ }^{12}$ Assuming a focal point has been shown to alleviate at least some coordination failures in Katz and Shapiro (1986). In our model, within-generation coordination would render the perfect substitutes case equivalent to the single-technology case.
} 
neously, but choosing incompatible technologies. For some parameter values, an enthusiast will delay adoption with positive probability $\left(\alpha_{h}^{*}<1\right)$.

\section{Results}

- From one to two technologies: Individual incentives. We now compare the adoption behaviour for one and two new technologies. Note first that if technology adoption can be coordinated by a social planner who observes the types of adopters, the second technology would clearly add more value. Any reduction in welfare is therefore due to the risk of splintering.

Proposition 2: In the two-technology case, a) the critical values for a $B E$ to obtain are higher for enthusiasts; and b) in a BE, the probability of adopting early is weakly lower for enthusiasts.

Proposition 2a states that an enthusiast will require $h$ to be higher in the two-technology case for her to adopt at $t=1$ compared to the onetechnology case. Hence, adding a second technology may kill off the market entirely. Consider a simple illustrative example: Suppose $a_{\max }^{x}=h=\widetilde{h}+\varepsilon$, which is sufficient to trigger adoption of $x$ in the one-technology case. In the presence of two technologies however, $h$ is below the critical value, i.e. $\widetilde{h}<h<\widehat{h}$, so that no adoption takes place at all. ${ }^{13,14}$ Proposition $2 \mathrm{~b}$ states that even within the range of a $B E$, an adopter's incentive to adopt early is weakly lower. The reason is that for some parameter ranges, $\alpha_{\mathbf{a}}^{*}$ is a nondegenerate mixed strategy (i.e. $0<\alpha_{\mathbf{a}}^{*}<1$ ) in the two-technology case, whereas adopting early is a dominant strategy with one technology if $a_{\max }$ exceeds the critical value for early adoption. With two technologies there may be an option value of waiting for biased and uniform enthusiasts, as we show in Proposition 1a. This means that the probability of early adoption is weakly decreasing with $a_{\min }$ for $a_{\max }=h$, i.e. $\alpha_{h}^{*}=\alpha_{h 0}^{*} \geq \alpha_{h l}^{*} \geq \alpha_{h h}^{*}$.

- From one to two technologies: Aggregate behaviour. We now

\footnotetext{
${ }^{13}$ We can construct an intermediate case with four types to obtain a more moderate decrease in expected adoption: Very high $\left(a_{\max } \gg \widetilde{h}\right)$, high $\left(a_{\max }=\widetilde{h}+\varepsilon\right), l$ and 0 . High types would drop out and overall expected adoption decreases.

${ }^{14}$ In a setting with no option to delay, this effect would not arise, although splintering may still occur.
} 
consider aggregate outcomes. First, we derive the probabilities of adoption outcomes in a $B E$, and we then calculate expected welfare.

\section{Unconditional Adoption Probabilities.}

Proposition 3: In a BE, introducing a second uncorrelated technology will imply: a) an increased likelihood of any adoption; b) a risk of splintering; c) a higher likelihood of single adopters of a technology for sufficiently high $q$ and low $p$; d) a higher likelihood of early adoption for sufficiently low $p$.

Proposition 3 outlines the effects of two new technologies if the timing of adoption is endogenous and adopters have the option not to switch. ${ }^{15}$ With more choice, some adoption is more likely (P3a) because there are more high-valuation adopters. However, in some instances adoption may result in splintering (P3b), a risk that does not exist with one new technology. In fact, for most parameter combinations (except for low $q$ and high $p$ ), the likelihood of single adopters adopting a technology is higher with two technologies (P3c). That is, higher adoption overall is not automatically a good sign as adopters may be orphaned and forego potential network effects. Regarding adoption timing, in the high-adoption $B E$ where $\alpha_{h 0}^{*}=\alpha_{h l}^{*}=$ $\alpha_{h h}^{*}=1$, early adoption is more likely with two technologies as a result of increased variety. This result is more nuanced for parameter ranges with relatively high $q$ and $p$ - in this case early adoption may be more likely in the one-technology case because the game resembles a coordination game (since there are hardly any intransigents) and adopters will try and mix adoption probabilities to avoid coordination failures, which would not be an issue in the one-technology case (P3d). We now examine how this affects aggregate expected welfare.

\section{Aggregate welfare.}

We focus on ex-ante aggregate expected welfare; that is, we consider the likelihood that two adopters of types $\mathbf{a}_{\mathbf{i}}$ and $\mathbf{a}_{\mathbf{j}}$ meet and calculate the

\footnotetext{
${ }^{15}$ Under exogenous timing and/or no option to remain with the incumbent technology, the risk of splintering remains, and may be exacerbated if timing is endogenous and all adopters switch eventually, as adopters may preempt each other by adopting early.
} 
resulting welfare from playing the game. For example, consider two adopters, one a stubborn enthusiast $(\mathbf{a}=(h 0))$, the other a uniform follower $(\mathbf{a}=(l l))$. The likelihood of this occurring is $\phi_{h 0} \phi_{l l}=p(1-p-q) \cdot q^{2}$, and the aggregate utility is $(h+b-c)+(l+b-c) .{ }^{16}$ Expected aggregate welfare is then the weighted sum of aggregate payoffs of all possible outcomes of the game. We generically write aggregate expected welfare as follows: ${ }^{17}$

$$
W=\sum_{i, j} \phi_{\mathbf{a}_{\mathbf{i}}} \phi_{\mathbf{a}_{\mathbf{j}}}\left(u_{\mathbf{a}_{\mathbf{i}}}+u_{\mathbf{a}_{\mathbf{j}}}\right)
$$

For the case with two perfect substitutes, we can show the following:

Proposition 4: Introducing an incompatible, perfectly substitutable technology is welfare-decreasing.

Here we find the intuition of our findings on individual adoption incentives confirmed: A second, undifferentiated technology is socially harmful. As we have shown previously, adoption may break down completely, and even if it does not, expected welfare is lower. In other words, having two similar versions of a technology can act as a 'spoiler'. These costs of incompatibility from splintering and the consequent loss in network benefits would exist in any situation with two new technologies, i.e. regardless of there being an option to remain with the old technology. Policymakers wanting to maximize expected welfare of a new technological generation will thus try to ensure that incompatible, but similar versions of the same product are not introduced in the market - policymakers could either mandate compatibility between the two variants (to eliminate the risk of splintering), or require a sufficient degree of heterogeneity between the technologies (to counteract the harmful 'coordination' effect).

We now turn to the case of two independently drawn technologies. The negative effect of a coordination failure with two incompatible technologies may be offset by a higher likelihood of an adopter being an enthusiast for one of the technologies. Since we are studying bandwagon equilibria, being an enthusiast implies that there is at least some probability of adopting early (see

\footnotetext{
${ }^{16}$ A stubborn enthusiast adopts at $t=1$ with certainty and a uniform follower adopts the previously adopted technology at $t=2$. This results in both adopters gaining network benefits $b$ on top of their net standalone benefits $h-c$ resp. $l-c$.

${ }^{17}$ The full expressions for expected aggregate welfare for the three cases (two uncorrelated technologies, single technology, two perfect substitutes) are in the Appendix.
} 
Proposition 1a). Proposition 5, illustrated in Figure 3, gives the conditions for the 'variety effect' to outweigh the 'coordination effect'.

Proposition 5: If $p$ is sufficiently high, two independent new technologies will lead to lower expected aggregate welfare than a single one. Conversely, if $p$ is sufficiently low, two technologies may be welfare superior.

An intuitive reading of this result goes as follows; in a situation where a single new technology is likely to generate a bandwagon anyway, offering potential adopters a second choice is harmful. This occurs when $p$, the likelihood of an adopter having a high valuation for any given new technology, is sufficiently high. Introducing a second new technology then increases the risk of orphaning à la Proposition 4. Conversely, if the likelihood of adopters being enthusiasts for any new technology is small (i.e. $p$ small) and the likelihood of being a follower is high (i.e. $q$ large), offering more variety is beneficial. That is, in cases where excess inertia is most prevalent (due to the high likelihood of adopters being followers), adding another new technology will improve expected welfare.

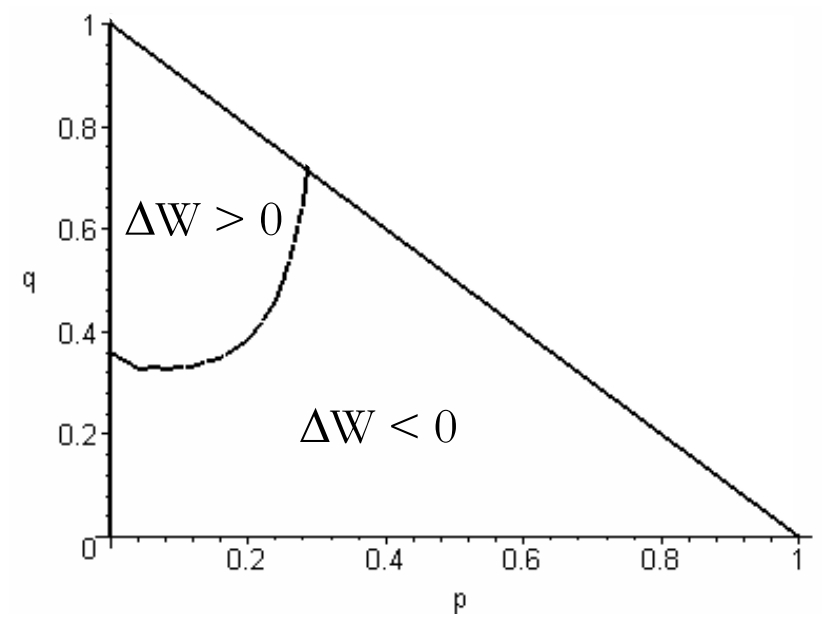

Figure 3: Parameter ranges for which adding an uncorrelated technology increases (decreases) aggregate welfare. (Note: Parameter values used (relative to $h$ ) were $l=.875 h, b=.5 h, c=1.35 h)$.

As we show in the Appendix, the parameter space in which adding a second technology increases expected welfare expands as $b, h, l$ rise and $c$ falls. 
This seems intuitive: Since the likelihood of a high valuation being drawn increases with two technologies, welfare increases if valuations are higher. Similarly, for high network effects, having a higher chance of starting a bandwagon (i.e. have two technologies on the market) is beneficial. Conversely, if an (unsuccessful) switch is more costly due to higher adoption cost, a higher risk of 'orphaned adoption' implies lower expected welfare.

\section{$5 \quad$ Limitations and Possible Extensions}

Although we believe that our model captures some interesting features of emerging network technologies, some limitations and suggestions for future work need to be addressed.

A-priori symmetry. Both new technologies are ex-ante symmetrical. That is, high, low, and zero valuations are identical for both technologies, as are network benefits and adoption costs. Changing any of these assumptions would obviously tip the game in the better technology's favour. If one technology was unanimously preferred by all adopters so that $l_{x}>h_{y}$, the coordination problem would not arise. If technologies are only ordered within the high and low valuations, i.e. $h_{x}>h_{y}$ and $l_{x}>l_{y}$, the coordination problem would be weaker, but it would still exist. An interesting case is where the distributions of valuations for different technologies differ. For example, if a technology with many prospective followers but few enthusiasts is introduced alongside one with more trendsetters but less followers, we may obtain a scenario where a switch to the 'mass-market' technology (higher $q$, lower $p$ ) may be efficient, but the 'trendy' technology is more likely to succeed. This also affects firm behaviour - for instance, Urban and von Hippel (1988) find that variants of a new computer-aided design (CAD) technology that have been designed to fit lead users' preferences have been comparatively more successful than ones that were not.

Competitively supplied technologies. We treat $c$ as an exogenous parameter in our model. If technologies are sponsored, however, prices are set endogeneously by firms. Katz and Shapiro (1986) show that a sponsored technology has an advantage over an unsponsored one even if it is inferior. They also show that with competition between two sponsored technologies, the technology which will be superior later on has an advantage. Their re- 
sults do not necessarily apply to our case however since they only model one old (superior today) and one new (superior tomorrow) technology. In our setting, increased competition within the new generation would bias the results in favour of the two-technology case. Even if the variety effect is moderate, having two sponsors heavily subsidizing adoptions in the first period in order to get a bandwagon started may make successful transition to a new technology more likely in the two-technology case than in the one-technology (monopoly) case.

'Large' adopters. In our model, adopters' decisions are directly interdependent. This is realistic in a setting where agents are firms, or where network effects are localized so consumers directly observe each other's decisions. Considering a model with adopters of measure zero would generate a process similar to diffusion models and adoption decisions would be based on aggregate adoption figures. ${ }^{18}$ Schmutzler (1998) develops a model of location choice with externalities in this spirit, and related empirical work by Koski (1999) and Berndt et al. (2003) estimates the impact of competition among multiple variants of a new product (PCs and Antiulcer drugs, respectively) on diffusion speed.

(In-)Compatibility. In our model, technologies are incompatible, which is the source of both inertia and splintering. Choosing compatibility between the new technologies would unambigously improve matters: As long as the loss in variety (by fixing some product characteristics to achieve compatibility) is not too severe, intra-generational compatibility is preferable. Backward compatibility would increase the valuation for each adopter by $\lambda b$, where $0 \leq \lambda \leq 1$ is the degree of backward compatibility. If this increase is uniform across adopters and technologies however, this would imply lower switching cost of $c-\lambda b$. If only one new technology is backward compatible (for example because it is owned by sponsor of the old technology), this will change the results as if there was vertical differentiation: If an adopter has equal standalone valuations for both technologies, the network benefit will cause him to prefer the backward compatible technology. Thus, if backward compatibility is important (i.e. $\lambda b$ large), this may result in a situation with one technology unanimously preferred, i.e. $l_{x}+\lambda b>h_{y}$.

\footnotetext{
${ }^{18}$ Adopters may still form expectations about the aggregate number of adoptions, but they expect the individual effect of their decision to be zero.
} 


\section{Conclusion and Implications}

The analysis of strategic technology adoption has largely focused on competition between two technologies, either a new and an old or two new ones. In this paper, we focus on the situation where potential adopters can choose between one of two new technologies or not adopt at all. We contrast our findings with the results by Farrell and Saloner (1985) on a single new technology and find that multiple technologies raise the threshold for any early adoption to occur. That is, an adopter must have a higher valuation for a new technology to find it worthwhile adopting in the presence of two new technologies. Further, we find that even if the valuation is high enough, adopters may still delay adoption. The first effect comes from the lower expected network benefit from adopting a technology, resulting in a higher critical value for adoption. The second effect emerges because high-valuation adopters play a coordination game; they want a bandwagon to emerge, but they would prefer someone else starting it to ensure compatibility by adopting the same. We also study the welfare effects of multiple technologies and find that although the probability of 'orphaning' increases compared to a single new technology, expected welfare with two technologies is higher for some parameter ranges. This is due to the variety effect - multiple imperfect substitutes on the market increase the probability that there will be a high-valuation adopter to start a bandwagon.

This paper is the first to theoretically analyze splintering with endogeneous adoption. In future work, it would be interesting to study past histories of technology introduction with multiple varieties to see which of the two effects identified in our model - the benefits from increased variety or the risk of splintering - dominates in specific cases. 


\section{A Proof of Propositions 1 - 5}

\section{A.1 Preliminaries}

\section{Equilibrium Conditions.}

Denote by $u_{S}, S \in\{S 1, S 2, S 3\}$ the expected net utilities in the three subgames at $t=2$, and $U_{t}, t=1,2$ the expected utilities from adopting early or delaying. Recall that $\phi_{\mathbf{a}}$ is the probability that an adopter is of type a. Further, define as $\rho_{1}$ the probability of early adoption of a specific technology (the probability of any early adoption is $\left.2 \rho_{1}\right), \rho_{2}$ the probability of late adoption in $S 1$, and $\rho_{F}$ the probability of an early adoption being followed. We now outline the conditions for a $B E$.

Subgames S2 and $S 3$ are straightforward. An adopter in $S 2$ (S3) will simply consider whether $u_{S 2}\left(u_{S 3}\right)>0$, i.e. $a_{\max }\left(a_{\min }\right)+b-c \gtrless 0$ and adopt accordingly.

$S 1$ requires updating expectations about the other adopter; player $i$ knows that $S 1$ could have been reached only if the other adopter would not adopt early with certainty, i.e. $\alpha_{\mathbf{a}_{j}}^{*}<1$. Therefore, $\beta_{\mathbf{a}}^{*}=1\left(\beta_{\mathbf{a}}^{*}=0\right)$ if $a_{\max }+\rho_{2} b>(\leq) c$.

We can now determine whether or not to adopt at $t=1$. In short, if $U_{1}>(<) U_{2}, \alpha_{\mathbf{a}}^{*}=1(0)$, and if $U_{1}=U_{2}, 0 \leq \alpha_{\mathbf{a}}^{*} \leq 1$ s.t. $U_{1} \geq 0$. Adopting at $t=1$ gives $a_{\max }-c+\left(\rho_{1}+\rho_{F}\right) b$. The final term is the network benefit times the probability of simultaneous adoption of the same technology at $t=1$ plus the probability of being followed at $t=2$. Delaying adoption gives an adopter flexibility to wait and observe which subgame is played at $t=2$, and react optimally in each subgame. The expected value from delaying therefore are the utilities in each subgame weighted by each subgame's likelihood of occurring. We therefore obtain the following condition for early adoption:

$$
a_{\max }+\left(\rho_{1}+\rho_{F}\right) b-c>\left(1-2 \rho_{1}\right) u_{S 1}+\rho_{1} u_{S 2}+\rho_{1} u_{S 3}
$$

To make the adoption probabilities $\rho$. more precise, we write:

$$
\begin{aligned}
\rho_{1} & =\sum \phi_{\mathbf{a}} \cdot \alpha_{\mathbf{a}}^{*} \\
\rho_{2} & =\frac{1}{2} \sum \phi_{\mathbf{a}} \cdot \max \left[0,\left(\beta_{\mathbf{a}}^{*}-\alpha_{\mathbf{a}}^{*}\right)\right] \\
\rho_{F} & =\frac{1}{2} \sum \phi_{\mathbf{a}} \cdot\left(\max \left[0,\left(\gamma_{\mathbf{a}}^{*}-\alpha_{\mathbf{a}}^{*}\right)\right]+\max \left[0,\left(\delta_{\mathbf{a}}^{*}-\alpha_{\mathbf{a}}^{*}\right)\right]\right)
\end{aligned}
$$


Lemma A1: For ex-ante symmetrical technologies, $\rho_{F} \geq \rho_{2}$.

Proof: $\beta_{\mathbf{a}} \geq 0$ implies that $a_{\max }+\rho_{2} b \geq c$. Since $\rho_{2} \leq 1, a_{\max }+b \geq$ $a_{\max }+\rho_{2} b$, so that $\gamma_{\mathbf{a}} \geq \beta_{\mathbf{a}}$. Therefore, the first term in large brackets in $\rho_{F}$, i.e. the number of "happy followers" - followers that end up adopting their preferred technology - is already at least as large as $\rho_{2}$, so that $\rho_{F} \geq \rho_{2}$.

Lemma A1 states that it is more likely to find followers for one's preferred technology than 'unprompted' adopters in 2. For ex-ante identical technologies, the total mass of adopters wanting to adopt in $S 1$ will split evenly among the technologies. The adopters ready to adopt their preferred technology regardless of previous adoption would adopt even more readily if guaranteed the network effect $\left(\rho_{F} \geq \rho_{2}\right)$.

Further, the following relations between expected utilities of different types hold in a $B E$ :

i) $\quad U_{1}(h 0)=U_{1}(h l)=U_{1}(h h) ; U_{2}(h 0)<U_{2}(h l)<U_{2}(h h)$

ii) $U_{1}(h 0)>u_{S 1}(h 0)$

iii) $U_{1}(l 0)=U_{1}(l l) ; U_{2}(l 0)<U_{2}(l l)$

iv) $u_{S 1}(l 0)=u_{S 1}(l l) ; u_{S 1}(h 0)=u_{S 1}(h l)=u_{S 1}(h h)$

i) states that the expected payoffs from early adoption is identical for all enthusiasts, but that the option value of waiting is increasing in the $a_{\min }$.

ii) implies that a stubborn enthusiast will always prefer adopting early to waitign and adopting later. This follows directly from Lemma A1.

iii) is similar to relation i) - the option value of waiting is higher for uniform followers than for biased followers.

iv) states that adopting in Subgame 1 will be equally profitable for all types with the same $a_{\max }$.

\section{A.2 Proof of Proposition 1a}

\section{A.2.1 Low-adoption BE}

We first characterize the low-adoption $B E$. Suppose that adopter $i$ (weakly) prefers technology $x$, i.e. $a^{x} \geq a^{y}$ for ease of exposition. We derive equilibrium strategies for each different type assuming that a $B E$ exists. We then derive the conditions for a $B E$ to exist.

- $\mathbf{a}=(\mathbf{0 0})$. An adopter of this type trivially will not adopt any technology, i.e. $\sigma_{00}^{*}=\{0,0,0,0\}$. 
- $\mathbf{a}=(\mathbf{h} \mathbf{0}) \cdot a_{\min }=0$, so that there is no incentive to delay, since $\rho_{F} \geq \rho_{2}$. Early adoption is assumed to be profitable (if it was not, it would not be for any type and consequently a $B E$ would not exist) so that $\mathbf{a}=(h 0)$ implies adoption at $t=1$, so that $\sigma_{h 0}^{*}=\{1,1,1,0\}$.

- $\mathbf{a}=(\mathbf{l 0})$ and $\mathbf{a}=(\mathbf{l l})$. In a $B E$, some types will be strict followers, i.e. they would never adopt unless the other adopter has. This implies that $U_{1}(l l), U_{1}(l 0), u_{S 1}(l l), u_{S 1}(l 0)<0$. Since both types have identical $u_{\max }=l$,they will have the same expected (negative) payoffs from adopting in $S 1$. Since $l+b>c$, we obtain the following strategies in a $B E: \sigma_{l 0}^{*}=\{0,0,1,0\}, \sigma_{l l}^{*}=\{0,0,1,1\}$.

- $\mathbf{a}=(\mathbf{h l})$ and $\mathbf{a}=(\mathbf{h h})$. Recall that $U_{1}(h 0)=U_{1}(h l)>0$. However, $u_{S 3}(h l)>0$ since $l+b>c$. It is therefore possible that $U_{1}(h l) \leq U_{2}(h l)$ although adopting early would be profitable. Suppose now that an $h l$ type is indifferent between adopting early and delaying, i.e. $U_{1}(h l)=$ $U_{2}(h l)$. This implies $U_{1}(h h)<U_{2}(h h)$ since $u_{S 3}(h l)<u_{S 3}(h h)$, so that $\alpha_{h h}^{*}=0$. In a $B E$ we have $\alpha_{l 0}^{*}=\alpha_{l l}^{*}=\beta_{l 0}^{*}=\beta_{l l}^{*}=0$. For an $h l$-type to be indifferent between adopting early or late, we need:

$$
h+\left(\frac{\rho_{1}}{2}+\rho_{F}\right) b-c=\left(\left(1-\frac{\rho_{1}}{2}\right) h+\frac{\rho_{1}}{2} l\right)+\left(\rho_{1}+\frac{\rho_{2}}{2}\right) b-c .
$$

Inserting the corresponding values for $\rho_{1}, \rho_{2}$, and $\rho_{F}$ and simplifying gives

$$
\alpha_{h l}^{*}=\frac{p(1-p-q)(h-l-b)+\left(\frac{p^{2}}{2}+q\right) b}{p q(h-l-b)-p q b} .
$$

The equilibrium strategies are then $\sigma_{h l}^{*}=\left\{\alpha_{h l}^{*}, 1,1,1\right\}$ and $\sigma_{h l}^{*}=\{0,1,1,1\}$.

\section{A.2.2 High-adoption BE}

- The equilibrium strategies for $a=(h 0),(l 0),(l l),(00)$ are derived analogously to the low-adoption case, i.e. $\sigma_{h 0}^{*}=\{1,1,1,0\}, \sigma_{l 0}^{*}=$ $\{0,0,1,0\}, \sigma_{l l}^{*}=\{0,0,1,1\}, \sigma_{00}^{*}=\{0,0,0,0\}$.

- $\mathbf{a}=(\mathbf{h l})$ and $\mathbf{a}=(\mathbf{h h})$. Suppose now that $U_{1}(h h)=U_{2}(h h)$ and consequently $U_{1}(h l)>U_{2}(h l)$. We know then that $\alpha_{h l}^{*}=\alpha_{h 0}^{*}=1$ and 
$\alpha_{l l}^{*}=\alpha_{l 0}^{*}=0$ and find the probability of adopting early by equating $U_{1}(h h)=U_{2}(h h)$ :

$$
h+\left(\frac{\rho_{1}}{2}+\rho_{F}\right) b-c=h+\left(\rho_{1}+\frac{\rho_{2}}{2}\right) b-c,
$$

which gives the following probability of adoption:

$$
\alpha_{h h}^{*}=\frac{\frac{3}{2} p^{2}+q-p q-p}{p^{2}}
$$

We can then characterize the strategies for $h l$ - and $h h$-types in the highadoption BE: $\sigma_{h l}^{*}=\{1,1,1,1\}, \sigma_{h h}^{*}=\left\{\alpha_{h h}, 1,1,1\right\}$.

The equilibrium strategies in the high-and low-adoption $B E$ therefore are:

\section{High-Adoption BE}

$$
\begin{aligned}
& \sigma_{h 0}^{*}=\{1,1,1,0\}, \sigma_{h l}^{*}=\{1,1,1,1\}, \sigma_{h h}^{*}=\left\{\alpha_{h h}, 1,1,1\right\}, \\
& \sigma_{l 0}^{*}=\{0,0,1,0\}, \sigma_{l l}^{*}=\{0,0,1,1\}, \sigma_{00}^{*}=\{0,0,0,0\} .
\end{aligned}
$$

\section{Low-Adoption BE}

$$
\begin{aligned}
& \sigma_{h 0}^{*}=\{1,1,1,0\}, \sigma_{h l}^{*}=\left\{\alpha_{h l}, 1,1,1\right\}, \sigma_{h h}^{*}=\{0,1,1,1\}, \\
& \sigma_{l 0}^{*}=\{0,0,1,0\}, \sigma_{l l}^{*}=\{0,0,1,1\}, \sigma_{00}^{*}=\{0,0,0,0\},
\end{aligned}
$$

where $\alpha_{h h}^{*}=\frac{\frac{3}{2} p^{2}-p q+q-p}{p^{2}}$, and $\alpha_{h l}^{*}=\frac{p(1-p-q)(l+b-h)-\left(\frac{p^{2}}{2}+q\right) b}{p q(l+b-h)+p q b}$.

\section{A.2.3 Critical Values.}

- $\mathbf{a}_{\max }=h$. Consider the high- and low-adoption $B E$. The critical values for early adoption to be profitable are the following:

$$
\begin{aligned}
\widehat{h}_{\text {high }} & =c-\left(p+q-p q-\frac{\alpha_{h h}}{2} p^{2}\right) b, \\
\widehat{h}_{\text {low }} & =c-\left(p+q-\alpha_{\text {hl }} p q\right) b, \\
\text { so that } \widehat{h}_{\text {high }} & >\widehat{h}_{\text {low }} .
\end{aligned}
$$

So $h>\widehat{h}_{h i g h}$ ensures that an $h$-type will consider adopting at $t=1$.

- $\mathbf{a}_{\max }=l$. We know that $u_{S 1}(l 0)=u_{S 2}(l l)$, so we can derive one critical value for both types. Adopting in Subgame $S 1$ is profitable if the 
probability that the other adopter will adopt as well is high enough conditional on her not being an early adopter. This gives us three scenarios, depending on whether $\alpha_{\mathbf{a}}=1$ or not. This is due to the fact that by observing no adoption at $t=1$ and moving to Subgame $S 1$ allows for Bayesian updating if $\alpha_{\mathbf{a}}=1$ - an adopter cannot be of a type that would have adopted with certainty at $t=1$ if $S 1$ is being played. We obtain the following critical values:

$$
\text { If }\left\{\begin{array}{c}
\alpha_{h 0}=\alpha_{h l}=\alpha_{h h}=1, \quad \widehat{l}_{h i g h 1}=c-\left(\frac{q^{2}+2 q(1-p-q)}{2\left(1-2 p\left(1-\frac{p}{2}\right)\right)}\right) b \\
\alpha_{h 0}=\alpha_{h l}=1, \alpha_{h h}<1, \quad \widehat{l}_{h i g h 2}=c-\left(\frac{p^{2}+q^{2}+2 q(1-p-q)}{2(1-2 p(1-p))}\right) b \\
\alpha_{h 0}=1, \alpha_{h l}, \alpha_{h h}<1, \quad \widehat{l}_{l o w}=c-\left(\frac{2 p q+p^{2}+q^{2}+2 q(1-p-q)}{2(1-2 p(1-p-q))}\right) b
\end{array}\right.
$$

It is easy to show that $\widehat{l}_{h i g h 1}<\widehat{l}_{h i g h 2}<\widehat{l}_{\text {low }}$. If $l<\widehat{l}_{\text {high } 1}, \beta_{l}=0$, which ensures the possibility of a $B E$.

\section{A.3 Proof of Proposition 1b}

As in the previous scenario, $\mathbf{a}=(0)$ will play $\sigma_{0}^{*}=\{0,0,0, \cdot\} \cdot{ }^{19}$

- $\mathbf{a}=(\mathbf{h})$. For enthusiasts, there is no incentive to delay adoption in a $B E$ because $\rho_{2}<\rho_{F}$, and only the highest type would attempt to start a bandwagon. If expected utility at $t=1$ is positive, an adopter with $\mathbf{a}=(h)$ will adopt early, i.e. $\alpha_{h}^{*}=1$. The condition for this is as follows:

$$
\begin{aligned}
h-c+(p+q) b & \geq 0, \text { i.e. } \\
h & \geq \widetilde{h} \equiv c-(p+q) b .
\end{aligned}
$$

- $\mathbf{a}=(\mathbf{l})$. Since in a $B E \mathbf{a}=(l)$ is the only candidate type for a follower, we derive the conditions for this to hold, i.e. $\sigma_{l}^{*}=\{0,0,1, \cdot\}$. Clearly, given $l-c+b>0, \gamma_{l}^{*}=1$. If there has been no adoption at $t=1(S 1)$, an adopter will update her belief about the other player. Knowing that

\footnotetext{
${ }^{19} \delta^{*}$ is irrelevant in the one-technology case.
} 
$a_{\max }<h$ for the other adopter, an adopter will only adopt at $t=2$ $\left(\beta_{l}^{*}=1\right)$ if the following inequality is met.

$$
\begin{aligned}
l-c+\left(\frac{q}{1-p}\right) b & \geq 0 \text {, i.e. } \\
\widetilde{\widetilde{l}} & \equiv c-\left(\frac{q}{1-p}\right) b>l .
\end{aligned}
$$

At $t=1$, we find that $l-c+(p+q) \geq l-c+\left(\frac{q}{1-p}\right) b$. Therefore, $\alpha_{l}^{*}=1(0)$ if $l>(\leq) \widetilde{l} \equiv c-(p+q) b$. Since $p+q>\frac{q}{1-p}, \widetilde{l}<\widetilde{\widetilde{l}}$, so that $\tilde{l}$ is the binding restriction for a $B E$ to emerge.

We can then characterize the $B E$ in the one-technology case as follows:

$$
\sigma_{h \cdot}^{*}=\{1,1,1, \cdot\}, \sigma_{l \cdot}^{*}=\{0,0,1, \cdot\}, \sigma_{0 \cdot}^{*}=\{0,0,0, \cdot\} .
$$

This is the unique $B E$ iff $h>\widetilde{h}, \widetilde{l}>l>c-b$, where $\widetilde{h}=\widetilde{l}=c-(p+q) b$.

\section{A.4 Proof of Proposition 1c}

First note that 0 -types will never adopt either technology, i.e. $\sigma_{0}^{*}=\{0,0,0,0\}$.

- $\mathbf{a}=(\mathbf{h})$. An $h$-type will consider adopting early with probability $\alpha_{h}$. Payoffs from adopting early or late are $U_{1}=h-c+\left(\frac{\alpha_{h}}{2} p+\left(1-\alpha_{h}\right) p+q\right) b$ and $U_{2}=h-c+\left(\alpha_{h} p+\frac{1-\alpha_{h}}{2} p\right) b$ respectively. Setting $U_{1}=U_{2}$ gives $\alpha_{h}^{*}=\frac{1}{2}+\frac{q}{p}$. Adopting early will then be profitable if $h>\bar{h}=$ $c-\left(\frac{3}{4} p+\frac{1}{2} q\right) b$.

- $\mathbf{a}=(\mathbf{l})$. As $l$-types are the only candidates for following, they play a bandwagon strategy if waiting is preferred to adopting early. This is given if $l<\bar{l}_{\text {high }}=c-(p+q) b$ for $\alpha_{h}^{*}<1$ and $l<\bar{l}_{\text {low }}=c-\left(\frac{q}{1-p}\right) b$ if $\alpha_{h}^{*}=1$.

Note that there are two different critical values $\bar{l}_{\text {high }}, \bar{l}_{\text {low }}$. These different values emerge because if $\alpha_{h}<1$, observing no adoption carries no information, i.e. the other adopter can still be an enthusiast playing a mixed strategy. In the other hand, if $\alpha_{h}=1$, observing no adoption rules out the 
possibility of the other adopter being an enthusiast. We summarize the $B E$ in the perfect substitutes case:

$$
\sigma_{h}^{*}=\left\{\alpha_{h}^{*}, 1,1,1\right\}, \sigma_{l}^{*}=\{0,0,1,1\}, \sigma_{0}^{*}=\{0,0,0,0\},
$$

where $\alpha_{h}^{*}=\frac{1}{2}+\frac{q}{p}$. A $B E$ occurs for the parameter ranges $h>\bar{h}, l<\bar{l}_{h i g h}$ if $\alpha_{h}^{*}=1$ and $l<\bar{l}_{\text {low }}$ if $\alpha_{h}^{*}<1$, where $\bar{h}=c-\left(\frac{3}{4} p+\frac{1}{2} q\right) b, \bar{l}_{\text {high }}=c-(p+q) b$, and $\bar{l}_{\text {low }}=c-\left(\frac{q}{1-p}\right) b$.

\section{A.5 Proof of Proposition 2}

\section{A.5.1 P2a)}

Consider the critical values for early adoption in our basic case and special case 1:

$$
\begin{aligned}
\widehat{h}_{\text {high }} & =c-\left(p+q-p q-\frac{\alpha_{h h}}{2} p^{2}\right) b \\
\widehat{h}_{\text {low }} & =c-\left(p+q-\alpha_{h l} p q\right) b \\
\widetilde{h} & \equiv c-(p+q) b
\end{aligned}
$$

From inspection of the expressions, it is easy to see that $\widehat{h}_{\text {low }}-\widetilde{h}=$ $\alpha_{\text {hl }} p q b \geq 0$. Since $\widehat{h}_{\text {high }}>\widehat{h}_{\text {low }}$, the critical value for adopting early in the two-technology case is higher than with a single new technology.

\section{A.5.2 P2b)}

In the case of a single new technology (Special case 1 ), $\sigma_{h}^{*}=\{1,1,1,0\}$. In the two-technology case, $\alpha_{h l}^{*}<1$ in some circumstances and $\alpha_{h h}^{*} \leq \alpha_{h l}^{*}$.

\section{A.6 Proof of Proposition 3}

We write $\widehat{\operatorname{Pr}} \bullet$ for probabilities referring to the two-technology case and $\widetilde{\operatorname{Pr}} \bullet$ for the one-technology scenario and $\Delta_{\bullet} \equiv\left(\widehat{\operatorname{Pr}}_{\bullet}-\widetilde{\operatorname{Pr}}_{\bullet}\right)$. 


\section{A.6.1 P3a)}

The likelihood of no adoption in the one- and two-technology scenarios are:

$$
\begin{aligned}
& \widehat{\operatorname{Pr}}_{\text {no switch }}=2 q(1-p-q)(1-p)^{2}+q^{2}(1-p)^{2}+(1-p-q)^{2}(1-p)^{2} \\
& \widetilde{\operatorname{Pr}}_{\text {no switch }}=q^{2}+2 q(1-p-q)+(1-p-q)^{2}
\end{aligned}
$$

For non-zero values of $p$ and $q, \Delta_{\text {no switch }}$ is always negative.

\section{A.6.2 P3b)}

The possibility of two adopters adopting different technologies simultaneously exists only for the two-technology case, so that $\Delta_{\text {splintering }}>0$.

\section{A.6.3 P3c)}

We show this result numerically. Figure 4 encloses the area where $\Delta_{\text {single adoption }}<$ 0 in the bottom right corner. Where $\Delta_{\text {early adoption }}<0$, the likelihood of single adopters is more likely with one new technology. We can see that this is the case for high values of $p$ and low values of $q$, and for sufficiently low values of $p(p<\underline{p} \approx .382)$, single adoption is more likely for any $q$.

\section{A.6.4 P3d)}

We show this result numerically. In Figure 5, we illustrate that for high values of $p(p>\underline{p}=.6)$ all permissible values of $q$ lead to lower adoption in the two-technology case, while for sufficiently low values of $p(p<p \approx .277)$ early adoption is higher for two technologies for all values of $q$.

\section{A.7 Proof of Proposition 4}

Expected welfare for a single new technology is

$$
W_{1}=2 p^{2}(h-c+b)+4 p q\left(\frac{h+l}{2}-c+b\right)+2 p(1-p-q)(h-c) .
$$

The expected welfare for two perfectly substitutable technologies is 


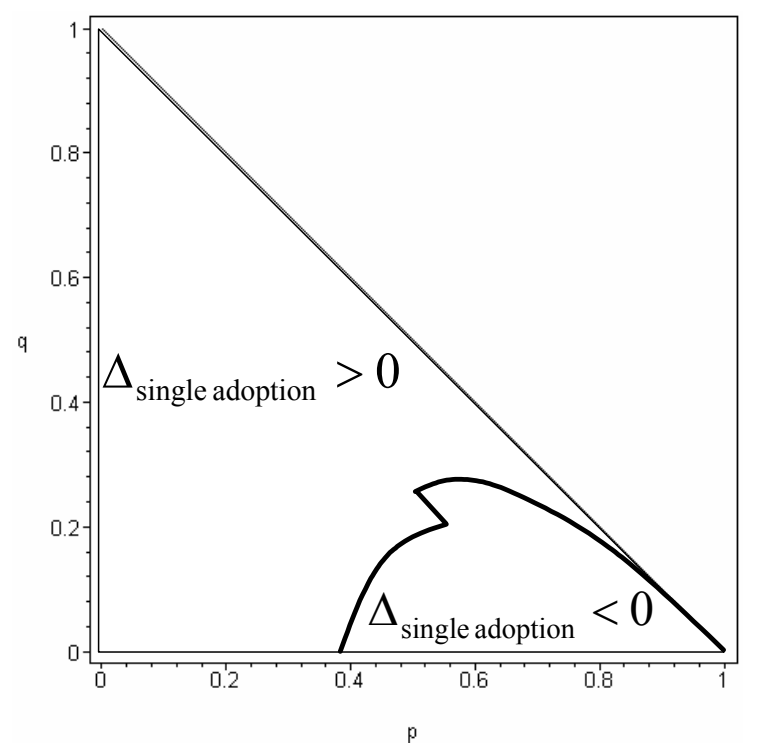

Figure 4: $\Delta_{\text {single adoption }} \lessgtr 0$ across permissible parameter ranges.

$$
\begin{aligned}
W_{S}= & 2 p^{2}\left(h-c+\left(\frac{1}{2}+\alpha_{h}^{*}\left(1-\alpha_{h}^{*}\right)\right) b\right)+ \\
& +2 p q\left(h-c+\alpha_{h}^{*}(l+2 b-c)\right)+2 p(1-p-q)(h-c) .
\end{aligned}
$$

Inspection reveals that for $\alpha_{h}^{*} \leq 1, \frac{1}{2}+\alpha_{h}^{*}\left(1-\alpha_{h}^{*}\right) \leq 1$. This implies that with two technologies, expected network benefits are strictly lower in a complete first-period switch and weakly lower in a bandwagon switch.

\section{A.8 Proof of Proposition 5}

Expected welfare for two technologies with uncorrelated valuations $W_{2}$ can be written as follows: 


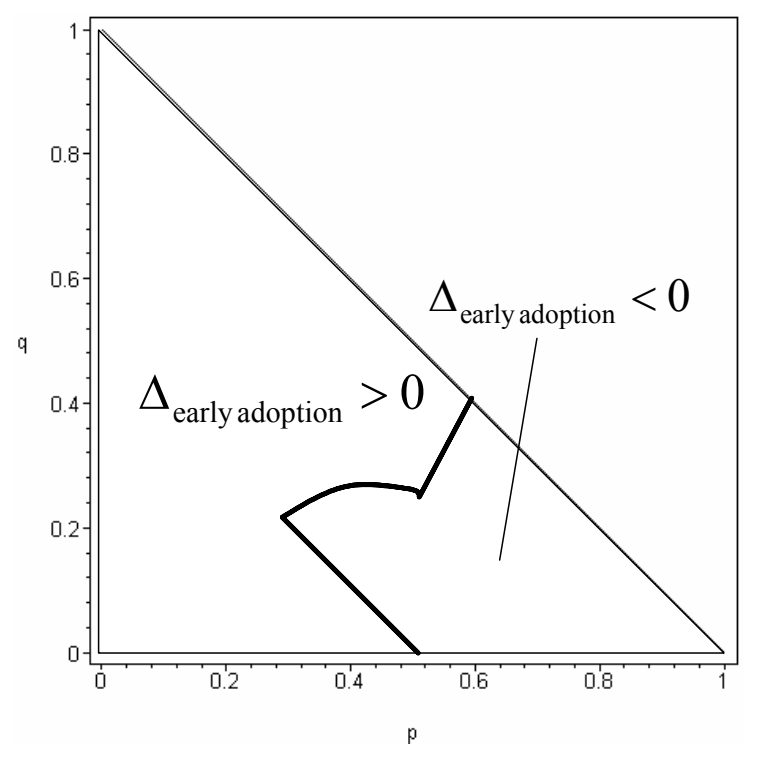

Figure 5: $\Delta_{\text {early adoption }} \lessgtr 0$ across permissible parameter ranges.

$$
\begin{aligned}
W_{2} & = \\
= & 2(h-c+b) p^{2}\left[2\left(1-\alpha_{h h}^{*} p\right)+p^{2}\left(3 \alpha_{h h}^{*}-\alpha_{h h}^{*^{2}}-\frac{3}{2}\right)-\right. \\
& \left.-2 p q\left(1-\alpha_{h h}^{*}-\alpha_{h l}^{*}+\alpha_{h h}^{*} \alpha_{h l}^{*}\right)\right]+ \\
& +(h+l-2 c+2 b) p\left[2(1-p)+2 q\left(1+\alpha_{h l}^{*}+2 q\left(1-\alpha_{h l}^{*}\right)\right)-\right. \\
& \left.-2 p q\left(3-p-q-\alpha_{h h}^{*}-\alpha_{h l}^{*}\left(2 p-\alpha_{h h}^{*} p-2+q-2 \alpha_{h l}^{*} q\right)\right)\right]+ \\
& +(h-c) p\left[4-p\left(6-\alpha_{h h}^{*} p-\alpha_{h h}^{*^{2}} p^{2}-\frac{5}{2} p^{2}+4 \alpha_{h h}^{*} p^{2}\right)+\right. \\
& +p q\left(5 \alpha_{h h}^{*} \alpha_{h l}^{*} p-3 \alpha_{h h}^{*} p+8 \alpha_{h l}^{*}-10 \alpha_{h l}^{*} p-6 p+2 q-8 \alpha_{h l}^{*} q+6 \alpha_{h l}^{*^{2}}-2 \alpha_{h h}^{*}\right)+ \\
& \left.+4 q\left(1+q-\alpha_{h l}^{*} q\right)\right]
\end{aligned}
$$

This expression cannot be analyzed analytically, and we show Proposition 5 numerically. Denote $\Delta W \equiv W_{2}-W_{1}$. As we are interested in parameter values for which expected welfare is higher (lower) with two independent technologies, we graph $\Delta W=0$ for our default values $h=1, l=.875$, $b=.5, c=1.35$ in $p, q$ space in Figure 3. We then discuss comparative static 
effects of changing our variables $h, l, b$ and $c$ in Figure 6 . Note that our results are scale-invariant, i.e. any value $\lambda$ used to scale all parameters $h, l$, $b$ and $c$ yields the same results.

To assess comparative statics, we change the variable in question by $5 \%$ and $10 \%$ and see how the parameter space with $\Delta W>0$ changes. We can see that it expands as $b, h, l$ rise and $c$ falls. Further, we can see that for a sufficiently large increase (resp. decrease in $c$ ) in each variable in question, any value of $q(\leq 1-p)$ and sufficiently low $p$ imply $\Delta W>0$.

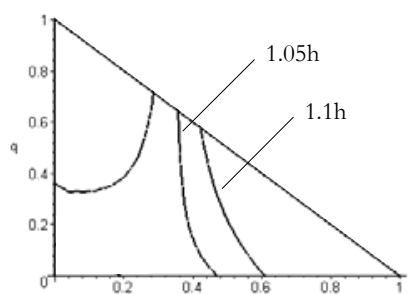

Increasing high valuation

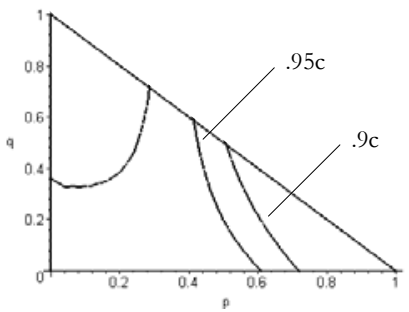

Decreasing costs

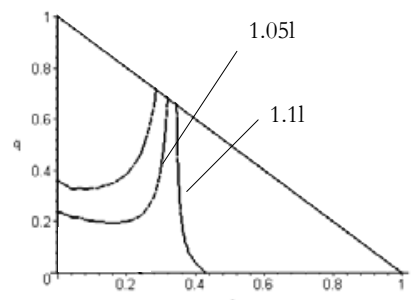

Increasing low valuation

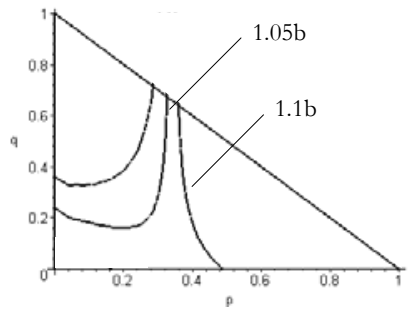

Increasing network benefits

Figure 6: Comparative Statics Analysis. 


\section{References}

[1] Arthur, W.B. (1989): Competing Technologies, Increasing Returns, and Lock-In by Historical Events. Economic Journal 99, 116-131.

[2] Augereau, A., S. Greenstein, M. Rysman (2004): Coordination vs. Differentiation in a Standards War: 56K Modems. Mimeo, Northwestern University.

[3] Berndt, E., R. Pindyck, P. Azoulay (2003): Consumption Externalities and Diffusion in Pharmaceutical Markets: Antiulcer Drugs. Journal of Industrial Economics 51, 243-270.

[4] Cabral, L.M. B., T. Kretschmer (2007): Standards Battles and Public Policy. In: S. Greenstein and V. Stango (eds.), Standards and Public Policy, Cambridge University Press, 329-344.

[5] Choi, J.P. (1994): Irreversible Choice of Uncertain Technologies with Network Externalities. Rand Journal of Economics 25, 382-401.

[6] Choi, J.P. (1997): Herd Behavior, the 'Penguin Effect', and the Suppression of Informational Diffusion: An Analysis of Informational Externalities and Payoff Interdependency, Rand Journal of Economics 28, 407-425.

[7] Choi, J.P., M. Thum (1998): Market Structure and the Timing of Technology Adoption with Network Externalities. European Economic Review 42, 225-244.

[8] Dranove, D., N, Gandal (2003): The DVD vs. DIVX Standard War: Empirical Evidence of Network Effects and Preannouncement Effects. Journal of Economics and Management Strategy 12, 363-386.

[9] Farrell, J., P. Klemperer (forthcoming): Coordination and Lock-In: Competition with Switching Costs and Network Effects. Forthcoming, Handbook of Industrial Organization, vol. 3.

[10] Farrell, J., G. Saloner (1985): Standardization, Compatibility, and Innovation. Rand Journal of Economics 16, 70-83.

[11] Katz, M., C. Shapiro (1986): Technology Adoption in the Presence of Network Externalities. Journal of Political Economy 94, 822-841. 
[12] Koski, H. (1999): The Installed Base Effect: Some Evidence from the Microcomputer Market. Economics of Innovation and New Technology 8, 273-310.

[13] Postrel, S. (1990): Competing Networks and proprietary standards: The case of quadraphonic sound. Journal of Industrial Economics 39, 169184.

[14] Saloner, G. (1990): Economic Issues in Computer Interface Standardization. Economics of Innovation and New Technology 1, 135-156.

[15] Schmutzler, A. (1998): Changing places - the role of heterogeneity and externalities in cumulative processes. International Journal of Industrial Organization 16, 445-461.

[16] Urban, G., E. von Hippel (1988): Lead User Analyses for the Development of New Industrial Products. Management Science 34, 569-582. 